\title{
Effects of a footwear intervention on foot pain and disability in people with gout: a randomised controlled trial
}

\author{
Mike Frecklington ${ }^{1 *} \mathbb{D}$, Nicola Dalbeth ${ }^{2,3}$, Peter McNair ${ }^{1}$, Trish Morpeth ${ }^{1}$, Alain C. Vandal ${ }^{4,5}$, Peter Gow ${ }^{6}$ and
} Keith Rome ${ }^{1}$

\begin{abstract}
Background: There is limited evidence supporting the long-term effect of a foot care package that includes footwear for people with gout. The aim of this study was to investigate the effectiveness of a footwear intervention on foot pain and disability in people with gout over 6 months.

Methods: Participants with gout $(n=94)$ were randomly allocated to either a control group (podiatric care and gout education) or footwear intervention group (podiatric care and gout education plus a commercially available athletic shoe). Measurements were undertaken at baseline and 2, 4, and 6 months. Primary outcome was foot pain severity. Secondary outcomes were overall pain, foot impairment/disability, footwear comfort, fit, ease and weight. Data were analysed using repeated measures models.

Results: Baseline foot pain scores were low, and no differences in foot pain scores were observed between groups over 6 months (adjusted effect estimate: $-6.7,95 \% \mathrm{Cl}-16.4$ to 2.9, $P=0.17$ ). Improvements between groups in overall pain scores (adjusted effect estimate: $-13.2,95 \% \mathrm{Cl}-22.2$ to $-4.3, P<0.01)$ and foot impairment/disability scores $(-4$. $7,95 \% \mathrm{Cl}-9.1$ to $-0.3, P=0.04$ ) favouring the footwear intervention were observed at 2 months, but not at 4 or 6 months. Improvements between groups in footwear fit (adjusted effect estimate: $-11.1,95 \% \mathrm{Cl}-21.1$ to $-1.0, P=0$. 03), ease $(-13.2,95 \% \mathrm{Cl}-23.8$ to $-2.7, P=0.01)$ and weight $(-10.3,95 \% \mathrm{Cl}-19.8$ to $-0.8, P=0.03)$ favouring the footwear intervention were also observed over 6 months. Similar improvements were observed for footwear comfort at 2 and 4 months. No other differences in secondary outcomes measured were observed at 6 months $(P>0.05)$.

Conclusions: Addition of footwear to a foot care package did not improve foot pain in people with gout. Short-term improvements in overall pain and foot impairment/disability and more durable improvements in footwear comfort and fit were observed with the footwear intervention.
\end{abstract}

Trial registration: ACTRN12614000209695. Registered 27 February 2014, http://www.anzctr.org.au/TrialSearch. aspx?searchTxt=ACTRN12614000209695\&isBasic=True

Keywords: Gout, Foot, Footwear, Foot pain

\footnotetext{
* Correspondence: mike.frecklington@aut.ac.nz

${ }^{1}$ Health and Rehabilitation Research Institute, AUT University, Private Bag

92006, Auckland 1142, New Zealand

Full list of author information is available at the end of the article
}

(c) The Author(s). 2019 Open Access This article is distributed under the terms of the Creative Commons Attribution 4.0 International License (http://creativecommons.org/licenses/by/4.0/), which permits unrestricted use, distribution, and reproduction in any medium, provided you give appropriate credit to the original author(s) and the source, provide a link to the Creative Commons license, and indicate if changes were made. The Creative Commons Public Domain Dedication waiver (http://creativecommons.org/publicdomain/zero/1.0/) applies to the data made available in this article, unless otherwise stated. 


\section{Background}

Gout commonly affects the articular and soft tissue structures of the feet, especially the first metatarsophalangeal joint [1] and Achilles tendon [2]. Foot problems are commonly described by people with gout [3], and people with gout experience high levels of foot pain, impairment and disability [4].

Regular podiatric care is associated with a reduction in foot pain and disability in people with inflammatory arthritis, including those with gout [5]. Prescribing footwear may be part of a foot care package for people with arthritis affecting the foot and ankle. For example, footwear interventions can improve foot pain and function in people with rheumatoid arthritis and foot osteoarthritis [6]. In rheumatoid arthritis, footwear interventions can also improve plantar pressure measurements and walking speed [6].

A footwear intervention may also benefit people with gout. A substantial proportion of people with gout wear footwear lacking in cushioning, support, stability and motion control [7]. Furthermore, patients with gout wearing poor footwear report higher pain and disability scores [7]. We have reported the results of a feasibility study, showing that commercially available athletic footwear with heel and forefoot cushioning, a dual density midsole and rocker sole reduces foot pain and disability in people with gout at 2 months [8].

Currently, the evidence supporting the long-term effect of a foot care package that includes footwear for people with gout is limited. The aim of this study was to investigate whether addition of footwear to a foot care package has benefit on foot pain and disability in people with gout over 6 months.

\section{Methods}

\section{Study design}

The study was a 6-month, two-arm, parallel randomised controlled trial comparing two foot care packages for people with gout, registered as a clinical trial with the Australian New Zealand Clinical Trials Registry (ACTR N12614000209695).

\section{Participants}

Participants were recruited from public hospital rheumatology clinics and through public newspaper advertising throughout Auckland, New Zealand. Participants were recruited between October 2014 and June 2016. Inclusion criteria were gout according to the 1977 preliminary American Rheumatism Association classification criteria [9] and over 20 years of age. Exclusion criteria were history of other inflammatory arthritis or neuromuscular disease, experiencing a gout flare at time of screening visit, medication for foot pain in the past 4 weeks, prescription of footwear and/or foot orthoses in the past 3 months, previous foot and ankle surgery or unable to walk $10 \mathrm{~m}$ unaided. The trial was approved by the New Zealand Ministry of Health, Health and Disability Ethics Committees (14/CEN/117), and all participants provided written informed consent.

\section{Randomisation and blinding}

Participants were allocated 1:1 to the control group (podiatric care and gout education) or footwear intervention group (podiatric care and gout education plus a commercially available athletic shoe) using unstratified block randomisation with random block sizes. Centralised randomisation allowed the use of a sealed opaque envelope system. Randomisation of participants was undertaken by a research assistant with sole access to envelopes and not involved in data collection. Treating clinicians were not involved in the randomisation of participants. Participants could not be blinded to their study group. Participants invited into the study were informed they would receive a foot care package, without specific mention of footwear. Post-randomisation, participants were not informed of the intervention modalities in the other randomisation group.

\section{Assessment}

Participants attended study visits at the Auckland University of Technology Podiatry Clinic from November 2014 to February 2017. Baseline assessment included the recording of age, gender, ethnicity, body mass index (BMI), medical history and current medications. Disease-specific data included latest serum urate, disease duration, number of gout flares in the last 3 months and tophus count (total and at the foot).

\section{Interventions}

Participants attended two-monthly visits over a 6-month period. At each study visit, participants received standardised podiatric care comprising of palliative care of nails and skin, temporary padding, wound care, emollient use, footwear advice, foot care advice and gout education delivered by an experienced podiatrist (TM). Gout education was delivered using a pamphlet produced by the New Zealand Ministry of Health including information on the causes of gout, the role of urate in development of gout, pharmacological management, monitoring of serum urate levels and general footwear advice (https://www.health. govt.nz/system/files/documents/topic_sheets/stop_gout_b ooklet-dec2015.pdf). General footwear advice included information regarding footwear comfort, fit, cushioning, sole and heel height. In addition, participants in the footwear intervention group received a pair of ASICS Cardio Zip 3 shoes to wear during daily activities (Fig. 1). This footwear was chosen based on the findings of a previous feasibility study [8], and its characteristics including heel/forefoot cushioning, dual density midsole, wide-fitting option and 


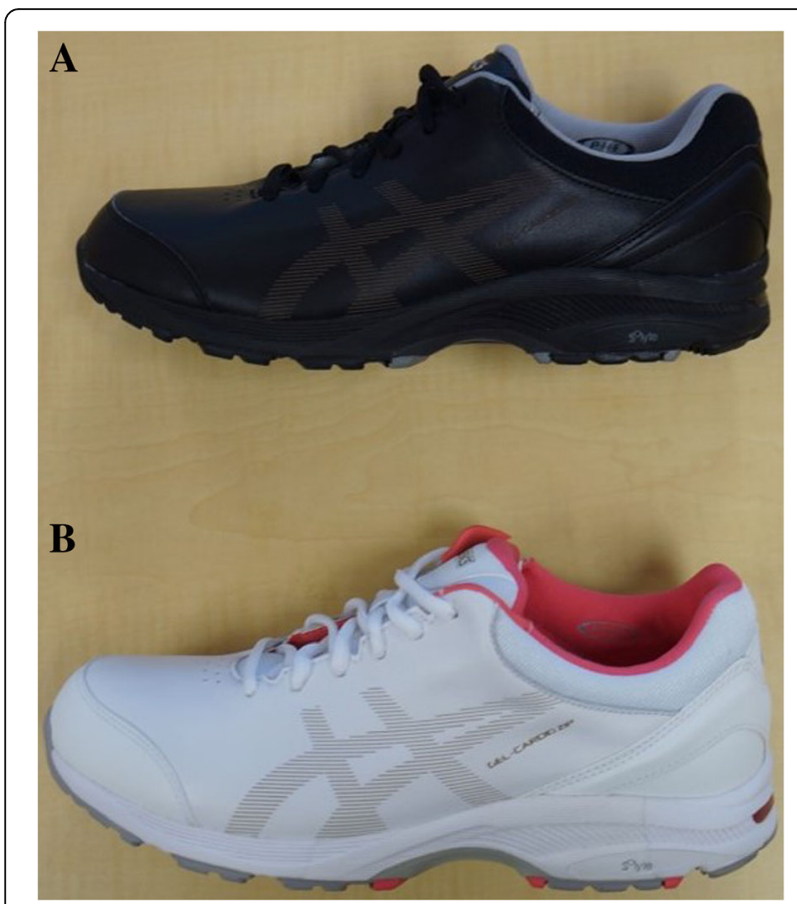

Fig. 1 ASICS Cardio Zip 3 shoes. a Men's and women's colour. b Women's colour only. Women had the option of choosing between the two colours

a zip for ease of fit. To determine the appropriate footwear size, the participant's foot length and width were measured by the podiatrist using a Brannock device. Women had the option of choosing between a black or white colour, with men having a black colour only. Footwear was then fitted by the podiatrist.

\section{Outcomes}

Outcome measures were measured at baseline and 2, 4, and 6 months. The primary outcome was participant-reported foot pain, measured using a $100-\mathrm{mm}$ visual analogue scale (VAS) using the anchors of 'no pain' $(0 \mathrm{~mm})$ to 'very severe pain' $(100 \mathrm{~mm})$ [4, 8]. Secondary outcome measures included participant-reported overall pain (global representation of pain) using 100-mm VAS, with lower scores indicating less pain, patient global assessment using 100-mm VAS, with lower scores indicating better wellbeing, and activity limitation using the Health Assessment Questionnaire (HAQ-II) [10]. Overall pain, patient global assessment and HAQ-II measures have been endorsed by Outcome Measures in Rheumatology (OMERACT) for use in gout studies [11]. Lower limb function was assessed using the activities of daily living and recreational activities subscales of the Lower Limb Tasks Questionnaire (LLTQ), with higher scores indicating better function [12]. Foot impairment and disability was measured using the Leeds Foot Impact Scale (LFIS), with lower scores indicating less impairment and disability [13]. The LLTQ and LFIS have been used in previous gout studies [8, 14]. Participant perceptions of footwear comfort, fit, ease and weight were each evaluated using $100 \mathrm{~mm}$ VAS, with lower scores indicating better comfort, fit, ease and weight. Self-reported footwear daily diaries were used to record footwear use and adverse events measured over the 6 months in the footwear intervention group, returned at each study visit [8]. The characteristics of the footwear worn by the control group were measured at each study visit. Participants were also asked at each visit about whether they had experienced a gout flare since the last study visit.

\section{Sample size}

Initial sample size calculations were based on a previous feasibility study [8]. To detect the minimally important difference of $-15 \mathrm{~mm}$ in the foot pain VAS $(-17.2 \mathrm{~mm}$ detected in feasibility study, $p=0.003$ ) with power 0.80 , using the repeated measures model detailed below with a baseline to 6-month correlation of 0.30 would require 52 participants in each group (correlation extrapolated from feasibility study). Using a conservatively estimated loss to follow-up rate of $25 \%$ at 6 months, the initial aim was to recruit 140 participants. A protocol amendment containing a revised sample size computation was submitted to and approved by an independent data monitoring committee, due to a decline in recruitment and lower than estimated withdrawal rates. The revision used a new estimated baseline to 6-month correlation of 0.49 in the primary outcome and a new dropout rate, based on the first 38 completions. A revised sample size of 39 completions per group was determined. At an estimated loss to follow-up rate of $15 \%$, the target recruitment was 92 participants (46 per group).

\section{Statistical analysis}

Primary and secondary analyses were based on an intention-to-treat (ITT) analysis set, from which only participants with no baseline nor post-randomisation data were excluded. Primary and other outcomes were compared across the treatment groups using repeated measures models of outcome data at 2, 4, and 6 months adjusted for baseline. A blind review of the data was undertaken at the end of the trial to consider the specific regression models to use, inclusion of covariates, the appropriateness of multiple imputations for any missing covariate and any necessary data transformation. Continuous outcomes were fitted using linear mixed models except for footwear-related visual analogue scale outcomes, fitted with a scaled zero-inflated beta regression model (generalised additive model for location, scale and shape) due to an increased proportion of zero scores recorded for these outcomes. Age, gender, ethnicity, BMI, colchicine use, non-steroidal anti-inflammatory drug use, prednisone 
use and the presence of subcutaneous tophi at baseline were considered for inclusion in the regression models during a blind review, absent all knowledge of allocation. Partial $R^{2}$ was used as the main selection criterion. Ten multiply imputed data sets were produced, using all observed data, under an assumption of Missingness at Random. No data transformation was found to be needed. No correction for multiple testing was applied. All tests were carried out at a significance level of 0.05 against two-sided alternatives. Data were analysed using SAS version 9.4 and $R$ version 3.2.

\section{Results}

\section{Participant flow and characteristics}

Figure 2 shows the flow of participants through the study. There were 187 potential participants screened and 94 randomised. Participants were predominantly male of New Zealand European ethnicity, with over 10 year's disease duration and on urate-lowering therapy (Table 1). High rates of obesity and comorbidities such as hypertension and cardiovascular disease were observed. Notable differences between groups included the higher number of tophi reported in the control group. Poor footwear was common at baseline, with the majority of participants wearing footwear which was worn and over 12 months old (Table 2).

Recorded protocol violations included randomisation of three participants who consented but did not complete the initial study visit (excluded from the ITT set), and the withdrawal of one participant who consented but was later found to have had foot surgery with a toe amputation, post-randomisation (included in the ITT set).

\begin{tabular}{|c|c|}
\hline \multicolumn{2}{|c|}{ Assessed for eligibility ( $n=187$ ) } \\
\hline & $\begin{array}{l}\text { Excluded after screening ( } n=93 \text { ) } \\
\text { - No gout diagnosis ( } n=8) \\
\text { - Diagnosis of other inflammatory arthritis ( } n=18) \\
\text { - Unable to speak English language }(n=7) \\
\text { - Previous foot/ankle surgery }(n=9) \\
\text { - Footwear/foot orthoses past } 3 \text { months }(n=1) \\
\text { - Medication for foot pain in past } 4 \text { weeks }(n=5) \\
\text { - Existing neuromuscular condition ( } n=17) \\
\text { - Current flare }(n=19) \\
\text { - Outside of study area }(n=9)\end{array}$ \\
\hline \multicolumn{2}{|c|}{ Randomised $(n=94)$} \\
\hline$\nabla$ & $\downarrow$ \\
\hline $\begin{array}{l}\text { Randomised to footwear intervention group ( } n=47) \\
\text { Received allocated intervention ( } n=45) \\
\text { Did not receive allocated intervention }(n=2) \\
\text { - Excluded for digital amputation }(n=1) \\
\text { - Did not attend study visit }(n=1)\end{array}$ & $\begin{array}{l}\text { Randomised to control group ( } n=47) \\
\text { Received allocated intervention ( } n=45) \\
\text { Did not receive allocated intervention ( } n=2) \\
\text { - Did not attend study visit }(n=2)\end{array}$ \\
\hline$\downarrow$ & $\nabla$ \\
\hline $\begin{array}{l}2 \text { month follow-up ( } n=40) \\
\text { Lost to follow up }(n=1) \\
\text { - Ill-fitting footwear ( } n=1) \\
\text { Did not attend study visit }(n=4)\end{array}$ & $\begin{array}{l}2 \text { month follow-up ( } n=39) \\
\text { Lost to follow up ( } n=2) \\
\text { - Deceased }(n=1) \\
\text { - Withdrew }(n=1) \\
\text { Did not attend study visit ( } n=4)\end{array}$ \\
\hline$\downarrow$ & $\downarrow$ \\
\hline $\begin{array}{l}4 \text { month follow-up ( } n=41 \text { ) } \\
\text { Did not attend study visit }(n=3 \text { ) }\end{array}$ & $\begin{array}{l}4 \text { month follow-up ( } n=37 \text { ) } \\
\text { Did not attend study visit ( } n=6 \text { ) }\end{array}$ \\
\hline$\downarrow$ & $\downarrow$ \\
\hline $\begin{array}{l}6 \text { month follow-up ( } n=43 \text { ) } \\
\text { - Did not attend study visit }(n=1)\end{array}$ & $\begin{array}{l}6 \text { month follow-up ( } n=42) \\
\text { Lost to follow-up }(n=1) \\
\text { - Withdrew }(n=1)\end{array}$ \\
\hline$\downarrow$ & $\downarrow$ \\
\hline $\begin{array}{l}\text { Analysed ( } n=46 \text { ) } \\
\text { Excluded from analysis (did not attend baseline } \\
\text { visit) }(n=1)\end{array}$ & $\begin{array}{l}\text { Analysed ( } n=45 \text { ) } \\
\text { Excluded from analysis (did not attend baseline } \\
\text { visit) }(n=2)\end{array}$ \\
\hline
\end{tabular}

Fig. 2 Flow of participants through the study 
Table 1 Baseline descriptive statistics

\begin{tabular}{|c|c|c|}
\hline Variable & Footwear intervention group $(n=47)$ & Control group $(n=47)$ \\
\hline Sex, male, $n(\%)$ & $40(85 \%)$ & $43(91 \%)$ \\
\hline Age, years & $62.6(17.0)$ & $62.4(13.7)$ \\
\hline $\mathrm{BMI}, \mathrm{kg} / \mathrm{m}^{2}$ & $30.2(6.4)$ & $32.0(7.0)$ \\
\hline \multicolumn{3}{|l|}{ Ethnicity, $n(\%)$} \\
\hline NZ European & $28(62 \%)$ & $26(57 \%)$ \\
\hline Pacific & $6(13 \%)$ & $11(23 \%)$ \\
\hline Asian & $7(16 \%)$ & $5(11 \%)$ \\
\hline Māori & $4(9 \%)$ & $4(9 \%)$ \\
\hline \multicolumn{3}{|l|}{ Gout history, mean (SD) } \\
\hline Disease duration (years) & $12.2(11.2)$ & $13.6(12.3)$ \\
\hline Flares prior 3 months & $0.7(0.9)$ & $0.4(0.7)$ \\
\hline Foot tophus, $n(\%)$ & $9(19 \%)$ & $17(36 \%)$ \\
\hline Any tophus, $n(\%)$ & $13(28 \%)$ & $24(51 \%)$ \\
\hline Serum urate, $\mathrm{mmol} / \mathrm{L}$ & $0.39(0.13)$ & $0.38(0.11)$ \\
\hline \multicolumn{3}{|l|}{ Medications, n (\%) } \\
\hline Urate-lowering therapy & $33(72 \%)$ & $30(64 \%)$ \\
\hline Colchicine & $15(33 \%)$ & $17(36 \%)$ \\
\hline Prednisone & $9(20 \%)$ & $10(21 \%)$ \\
\hline NSAID & $12(27 \%)$ & $13(29 \%)$ \\
\hline Diuretic & $8(18 \%)$ & $5(11 \%)$ \\
\hline \multicolumn{3}{|l|}{ Medical History, $n$ (\%) } \\
\hline Hypertension & $22(48 \%)$ & $22(54 \%)$ \\
\hline Cardiovascular disease & $13(28 \%)$ & $11(24 \%)$ \\
\hline Type 2 diabetes & $7(15 \%)$ & $3(7 \%)$ \\
\hline Peripheral vascular disease & $4(9 \%)$ & $3(7 \%)$ \\
\hline Peripheral neuropathy & $3(7 \%)$ & $5(11 \%)$ \\
\hline
\end{tabular}

$B M I$ body mass index, NSAID non-steroidal anti-inflammatory drug

Four participants in the footwear intervention group were unable to wear their allocated footwear due to discomfort. Of these participants, three remained enrolled in the trial and continued to receive the other facets of their allocated intervention with one participant withdrawal. Participants in the footwear intervention group reported wearing their allocated footwear on average $24 \mathrm{~h}$ per week during the study period. Participants in the control group continued to wear footwear of similar type, age and wear during the study period (Additional file 1). At the 6-month follow-up, $89 \%$ of the control group and $91 \%$ of the footwear intervention group completed the study.

\section{Primary outcome}

All efficacy endpoints and covariate adjustments are shown in Table 3. Baseline foot pain scores were low. There was no difference in foot pain at any time-point over the 6-month study period between the two groups (adjusted effect estimate: $-6.7,95 \% \mathrm{CI}-16.4$ to $2.9, P=0.17$ ).

\section{Secondary outcomes}

Improvements between groups in overall pain scores (adjusted effect estimate: $-13.2,95 \% \mathrm{CI}-22.2$ to $-4.3, P<$ 0.01 ) favouring the footwear intervention were observed at 2 months, but there was no difference between the groups at 4 or 6 months (adjusted effect estimate at 6 months: 4.0, $95 \% \mathrm{CI}-13.6$ to $5.7, P=0.42$ ). Foot-related impairment and disability was reduced at 2 months in the footwear intervention group (adjusted effect estimate: -4.7 , 95\% CI -9.7 to $-0.3, P=0.04)$, but there was no difference between groups at 4 or 6 months (adjusted effect estimate at 6 months: $-3.0,95 \% \mathrm{CI} 0.2$ to $-1.8, P=0.21$ ). No between-group differences in patient global assessment, HAQ-II and LLTQ were observed (Table 2).

Between-group differences favouring the footwear intervention were observed in footwear comfort at 
Table 2 Baseline footwear characteristics

\begin{tabular}{|c|c|c|}
\hline Variable & Footwear intervention group $(n=47)$ & Control group $(n=47)$ \\
\hline \multicolumn{3}{|c|}{ Footwear type, $n$ (\%) } \\
\hline Good & $23(51 \%)$ & $23(51 \%)$ \\
\hline Athletic & 5 & 11 \\
\hline Oxford & 10 & 5 \\
\hline Therapeutic & 2 & 0 \\
\hline Walking & 6 & 7 \\
\hline Moderate & $3(7 \%)$ & $2(4 \%)$ \\
\hline Boot & 3 & 2 \\
\hline Poor & $19(42 \%)$ & $20(44 \%)$ \\
\hline Sandal & 8 & 4 \\
\hline Moccasin & 4 & 7 \\
\hline Flip-flop & 4 & 4 \\
\hline Slipper & 2 & 4 \\
\hline Court & 1 & 0 \\
\hline Mule & 0 & 1 \\
\hline \multicolumn{3}{|c|}{ Footwear age, $n(\%)$} \\
\hline$<6$ months & $8(18 \%)$ & $12(27 \%)$ \\
\hline $6-12$ months & $5(11 \%)$ & $9(20 \%)$ \\
\hline$>12$ months & $31(70 \%)$ & $24(53 \%)$ \\
\hline \multicolumn{3}{|c|}{ Upper wear, $n(\%)$} \\
\hline Neutral & $34(77 \%)$ & $26(58 \%)$ \\
\hline Medial & $9(20 \%)$ & $17(38 \%)$ \\
\hline Lateral & $1(2 \%)$ & $2(4 \%)$ \\
\hline \multicolumn{3}{|c|}{ Midsole wear, n (\%) } \\
\hline Neutral & $38(86 \%)$ & 31 (69\%) \\
\hline Medial & $9(20 \%)$ & $9(20 \%)$ \\
\hline Lateral & $1(2 \%)$ & $5(11 \%)$ \\
\hline \multicolumn{3}{|c|}{ Outsole wear, $n(\%)$} \\
\hline None & $2(4 \%)$ & $7(16 \%)$ \\
\hline Partly worn & 35 (78\%) & $29(64 \%)$ \\
\hline Fully worn & $8(18 \%)$ & $9(20 \%)$ \\
\hline \multicolumn{3}{|c|}{ Outsole wear pattern, $n(\%)$} \\
\hline None & $1(2 \%)$ & $6(13 \%)$ \\
\hline Normal & $17(38 \%)$ & $19(42 \%)$ \\
\hline Medial & $0(0 \%)$ & $0(0 \%)$ \\
\hline Lateral & $27(60 \%)$ & $20(44 \%)$ \\
\hline
\end{tabular}

2 months (adjusted effect estimate: -10.4 , 95\% CI -19.9 to -0.9, $P=0.03$ ) and 4 months (adjusted effect estimate: $-11.3,95 \% \mathrm{CI}-21.4$ to $-1.3, P=0.03$ ), but not at 6 months (adjusted effect estimate: $-8.0,95 \%$ CI -19.2 to 3.3 , $P=0.16$ ). Similarly, between-group differences favouring the footwear intervention were observed in footwear fit (adjusted effect estimate: $-11.1,95 \%$ CI -23.0 to -1.0 , $P=0.03$ ), footwear ease (adjusted effect estimate: -13.2 , $95 \% \mathrm{CI}-23.8$ to $-2.7, P=0.01$ ) and footwear weight (adjusted effect estimate: -10.3 , 95\% CI -19.8 to -0.8 , $P=0.03)$ at all time-points over the 6-month study period.

\section{Adverse events}

Two participants (4\%) in the footwear intervention group developed foot blisters and one participant (1\%) in the footwear intervention group withdrew from the study due to footwear discomfort. During the trial period, 16 participants (34\%) in the control group and 
Table 3 Outcome measure scores and effect estimates adjusted from baseline

\begin{tabular}{|c|c|c|c|c|}
\hline & Footwear intervention group mean (SD) & Control group mean (SD) & Adjusted effect estimate & $P$ \\
\hline \multicolumn{5}{|c|}{ Foot pain VAS } \\
\hline Baseline & $14.8(18.7)$ & $17.5(22.4)$ & & \\
\hline 2 months & $10.7(13.0)$ & $16.8(21.8)$ & $-5.0(-12.9$ to 2.8$)$ & 0.21 \\
\hline 4 months & $13.8(23.0)$ & $16.1(22.3)$ & $-1.8(-10.1$ to 6.4$)$ & 0.66 \\
\hline 6 months & $13.1(20.8)$ & $20.5(26.1)$ & $-6.7(-16.4$ to 2.9$)$ & 0.17 \\
\hline \multicolumn{5}{|c|}{ Overall pain VAS } \\
\hline Baseline & $18.7(19.6)$ & $17.7(23.9)$ & & \\
\hline 2 months & $9.7(13.6)$ & $23.3(27.5)$ & $-13.2(-22.2$ to -4.3$)$ & $<0.01$ \\
\hline 4 months & $16.2(19.4)$ & $17.9(22.8)$ & $-2.3(-0.5$ to 0.6$)$ & 0.65 \\
\hline 6 months & $16.3(19.2)$ & $20.7(26.8)$ & $-4.0(-13.6$ to 5.7$)$ & 0.42 \\
\hline \multicolumn{5}{|c|}{ Patient Global Assessment VAS ${ }^{a}$} \\
\hline Baseline & $22.7(24.5)$ & $21.5(25.8)$ & & \\
\hline 2 months & $17.7(24.2)$ & $16.4(21.6)$ & $1.2(-7.4$ to 9.9$)$ & 0.78 \\
\hline 4 months & $14.6(16.6)$ & $16.6(20.2)$ & $-2.8(-11.9$ to 6.3$)$ & 0.55 \\
\hline 6 months & $15.3(19.4)$ & $18.8(21.9)$ & $-3.4(-12.6$ to 5.7$)$ & 0.46 \\
\hline \multicolumn{5}{|c|}{ Health Assessment Questionnaire II } \\
\hline Baseline & $0.5(0.6)$ & $0.4(0.5)$ & & \\
\hline 2 months & $0.5(0.6)$ & $0.4(0.4)$ & $-0.1(-0.3$ to 0.1$)$ & 0.36 \\
\hline 4 months & $0.6(0.6)$ & $0.3(0.5)$ & $0.0(-0.2$ to 0.2$)$ & 0.84 \\
\hline 6 months & $0.5(0.5)$ & $0.4(0.6)$ & $-0.1(-0.3$ to 0.1$)$ & 0.28 \\
\hline \multicolumn{5}{|c|}{ LFIS total score } \\
\hline Baseline & $15.5(11.5)$ & $15.4(12.5)$ & & \\
\hline 2 months & $13.8(13.0)$ & $16.4(14.1)$ & $-4.7(-9.1$ to -0.3$)$ & 0.04 \\
\hline 4 months & $14.9(14.2)$ & $14.2(12.3)$ & $-1.3(-6.1$ to 3.5$)$ & 0.59 \\
\hline 6 months & $14.4(13.6)$ & $16.9(14.2)$ & $-3.0(0.2$ to 1.8$)$ & 0.21 \\
\hline \multicolumn{5}{|c|}{ LLTQ activities of daily I } \\
\hline Baseline & $32.7(8.2)$ & $33.8(6.8)$ & & \\
\hline 2 months & $34.8(7.2)$ & $32.9(8.0)$ & $2.2(-0.2$ to 4.6$)$ & 0.07 \\
\hline 4 months & $32.9(8.1)$ & $35.4(6.7)$ & $-0.4(-3.1$ to 2.3$)$ & 0.77 \\
\hline 6 months & $34.0(6.9)$ & $33.8(7.7)$ & $1.1(-1.2$ to 3.4$)$ & 0.35 \\
\hline \multicolumn{5}{|c|}{ LLTQ recreational activities ${ }^{b}$} \\
\hline Baseline & $22.7(11.8)$ & $21.1(11.6)$ & & \\
\hline 2 months & $23.5(14.2)$ & $22.8(11.5)$ & $0.8(-2.8$ to 4.4$)$ & 0.66 \\
\hline 4 months & $20.8(12.8)$ & $25.1(9.9)$ & $-3.4(-7.5$ to 0.8$)$ & 0.11 \\
\hline 6 months & $21.7(12.6)$ & $22.2(12.0)$ & $-0.9(-4.8$ to 3.0$)$ & 0.66 \\
\hline \multicolumn{5}{|c|}{ Footwear comfort VAS } \\
\hline Baseline & $24.0(21.9)$ & $27.6(28.0)$ & & \\
\hline 2 months & $10.3(13.1)$ & $26.2(26.5)$ & $-10.4(-19.9$ to -0.9$)$ & 0.03 \\
\hline 4 months & $9.1(9.8)$ & $24.0(21.0)$ & $-11.3(-21.4$ to -1.3$)$ & 0.03 \\
\hline 6 months & $17.5(23.5)$ & $27.9(28.4)$ & $-8.0(-19.2$ to 3.3$)$ & 0.16 \\
\hline \multicolumn{5}{|c|}{ Footwear fit VAS ${ }^{d}$} \\
\hline Baseline & $20.6(20.1)$ & $24.0(27.2)$ & & \\
\hline 2 months & $9.8(16.0)$ & $22.2(21.3)$ & $-9.5(-17.2$ to -1.8$)$ & 0.02 \\
\hline 4 months & $10.3(13.6)$ & $22.2(20.4)$ & $-11.1(-19.9$ to -2.4$)$ & 0.01 \\
\hline
\end{tabular}


Table 3 Outcome measure scores and effect estimates adjusted from baseline (Continued)

\begin{tabular}{|c|c|c|c|c|}
\hline & Footwear intervention group mean (SD) & Control group mean (SD) & Adjusted effect estimate & $P$ \\
\hline 6 months & $11.9(20.0)$ & $27.9(28.4)$ & $-11.1(-21.1$ to -1.0$)$ & 0.03 \\
\hline \multicolumn{5}{|c|}{ Footwear ease VAS } \\
\hline Baseline & $20.9(23.0)$ & $19.3(23.8)$ & & \\
\hline 2 months & $12.7(19.1)$ & $26.8(28.2)$ & $-9.8(-19.4$ to -0.3$)$ & 0.04 \\
\hline 4 months & $10.2(16.6)$ & $23.8(25.2)$ & $-12.3(-23.0$ to -1.6$)$ & 0.02 \\
\hline 6 months & $11.3(19.7)$ & $27.9(28.4)$ & $-13.2(-23.8$ to -2.7$)$ & 0.01 \\
\hline \multicolumn{5}{|c|}{ Footwear weight VAS ${ }^{d}$} \\
\hline Baseline & $21.9(21.9)$ & $22.7(24.6)$ & & \\
\hline 2 months & $12.7(17.8)$ & $27.0(26.6)$ & $-9.7(-19.5$ to 0.0$)$ & 0.05 \\
\hline 4 months & $13.6(20.3)$ & $24.6(20.4)$ & $-10.8(-20.6$ to -0.9$)$ & 0.03 \\
\hline 6 months & $11.4(19.7)$ & $27.9(28.4)$ & $-10.3(-19.8$ to -0.8$)$ & 0.03 \\
\hline
\end{tabular}

VAS visual analogue scale, LFIS Leeds Foot Impact Scale, LLTQ Lower Limb Tasks Questionnaire

${ }^{\mathrm{a} B M I}$ adjusted

${ }^{\mathrm{b}}$ Age adjusted

${ }^{\mathrm{C}} \mathrm{BMI}$ and prednisone adjusted

${ }^{\mathrm{d}}$ Sex and BMI adjusted

eEthnicity and BMI adjusted

Data in italics indicates statistical significance

14 participants $(30 \%)$ in the footwear intervention group experienced a gout flare.

\section{Discussion}

This is the first randomised controlled trial of a podiatric intervention in gout. Although improvements in footwear comfort, fit and ease were observed in the footwear intervention group throughout the study period, no significant difference in foot pain was observed between groups. Short-term improvements in both overall pain and foot-related impairment and disability favouring the footwear intervention group were observed at the two-month time-point, consistent with the previous feasibility study [8].

The low levels of foot pain at the time of the baseline visit may have contributed to a floor effect, suggesting that clinical meaningful changes in foot pain could not be detected. Foot pain was not part of the inclusion criteria based on the previous feasibility study [8], which may have also contributed to the baseline foot pain levels observed. This highlights the challenge of studying pain as an outcome in gout which is an intermittently flaring condition. We observed baseline serum urate levels were close to target guidelines [15] and participants reported a low number of flares in the 3 months prior to the trial, which suggests generally well-controlled disease. Our findings for baseline foot pain levels were lower than the previous feasibility study [8], however, were consistent with previous studies measuring foot pain in people with longstanding gout during an intercritical period $[4,15]$.

Comfort and fit have been identified as important factors in footwear selection for people with gout [7]. Footwear is an important concern for people with gout, who often describe difficulty finding suitable footwear [16]. Improvements in footwear comfort, fit, weight and ease were observed in the footwear intervention group. The footwear received by the footwear intervention group had a number of characteristics which have been identified as beneficial for people with gout when compared to participants own footwear [8]. Footwear characteristics including correct footwear fit, the presence of cushioning and good torsional stiffness have previously been identified as influencers of subjective footwear comfort [17]. In the footwear intervention group, the fitting of footwear by a clinician may also be a factor. The footwear habits of the control group did alter during the trial, despite the footwear advice delivered. This furthers highlight the challenges that people with gout have finding appropriate footwear $[16,18]$. These findings suggest that helping people with gout find footwear with good characteristics is important; however, this may not involve the need for expensive footwear prescription.

Strengths of this study include the use of OMERACT-endorsed patient-reported outcomes for gout [11], high retention rates in both groups, and novelty as the first randomised controlled trial of a podiatric intervention in people with gout. The key study limitation was that participants could not be blinded to the footwear intervention, which may have biased the study outcomes, as all endpoints were patient-reported. We did attempt to reduce this bias by informing participants that they would be receiving a foot care package without the specific mention of receiving footwear and ensuring that all participants received a comprehensive foot care intervention. The study was undertaken in an urban New Zealand city and the findings may lack generalisability to other settings. The footwear 
used in this study had distinct characteristics, so it is unclear whether these findings can be generalised to other types of footwear such as open-toed footwear.

This study has focused on a commercially available athletic shoe. The podiatric care package was limited to standardised care and foot health advice, with or without the footwear intervention, and the role of other interventions such as foot orthoses is unknown. Further investigation into other footwear interventions for people with gout, including cost-effectiveness, is warranted. Changes to structural properties of the footwear through use may have also been a potential reason that long-term benefits were not observed. The effects of wear on the structural properties of footwear over time, and its relationship with biomechanical parameters such as plantar pressure and patient-reported outcomes such as foot pain, impairment and disability, are unknown. Future work might also explore factors which influence foot care and footwear use, and the willingness to pay for appropriate footwear.

\section{Conclusions}

The footwear intervention did not significantly improve foot pain in people without high baseline levels of foot pain. However, short-term improvements in overall pain and foot impairment/disability, and more durable improvements in footwear comfort and fit were observed with the footwear intervention.

\section{Additional file}

Additional file 1: Control group footwear characteristics. (DOCX $16 \mathrm{~kb}$ )

\section{Abbreviations}

BMI: Body mass index; HAQ-II: Health Assessment Questionnaire; ITT: Intention-to-treat; LFIS: Leeds Foot Impact Scale; LLTQ: Lower Limb Tasks Questionnaire; OMERACT: Outcome Measures in Rheumatology; VAS: visual analogue scale

\section{Acknowledgements}

We thank Katie Hayden and Paul Tan for their assistance with recruiting participants into the study, Heidi Barton (Podiatrist) for assistance with data collection, Ben Elliot for assistance with data management and Emma Witt and Janet Pearson for their involvement in data monitoring.

\section{Funding}

This work was supported by the Auckland Medical Research Foundation [grant number 5114003].

\section{Availability of data and materials}

Data and material available for this study would require further approval upon request from the corresponding author.

\footnotetext{
Authors' contributions

MF had full access to all of the study data and takes responsibility for the integrity and accuracy of the statistical analysis. MF, ND, PMcN, AV, PG and KR contributed to the study design, data analyses, data interpretation and manuscript preparation. TM contributed to acquisition of data and manuscript preparation. All authors were involved in the drafting of the manuscript, with all authors approving the final version to be submitted for publication.
}

\section{Ethics approval and consent to participate}

The trial was approved by the New Zealand Ministry of Health, Health and Disability Ethics Committees (14/CEN/117) and all participants provided written informed consent.

\section{Consent for publication}

Not applicable.

\section{Competing interests}

N. D. has received consulting fees, speaker fees or grants from Takeda, Teijin, Menarini, Amgen, Ardea Biosciences, AstraZeneca, Horizon, Cymabay and Kowa, outside the submitted work. K. R. has received funding from ASICS, outside the submitted work. ASICS had no role in the design of the study. The footwear used in the study was purchased at retail price. The other authors declare that they have no competing interests.

\section{Publisher's Note}

Springer Nature remains neutral with regard to jurisdictional claims in published maps and institutional affiliations.

\section{Author details}

${ }^{1}$ Health and Rehabilitation Research Institute, AUT University, Private Bag 92006, Auckland 1142, New Zealand. ²Department of Medicine, The University of Auckland, Auckland, New Zealand. ${ }^{3}$ Auckland District Health Board, Auckland, New Zealand. ${ }^{4}$ Department of Biostatistics \& Epidemiology, AUT University, Auckland, New Zealand. ${ }^{5}$ Research \& Evaluation Office, Ko Awatea, Counties Manukau Health, Auckland, New Zealand. ${ }^{6}$ Counties Manukau District Health Board, Auckland, New Zealand.

Received: 5 December 2018 Accepted: 1 April 2019

Published online: 24 April 2019

\section{References}

1. Stewart S, Dalbeth N, Vandal AC, Rome K. The first metatarsophalangeal joint in gout: a systematic review and meta-analysis. BMC Musculoskelet Disord. 2016:17:69.

2. Dalbeth N, Kalluru R, Aati O, Horne A, Doyle AJ, McQueen FM. Tendon involvement in the feet of patients with gout: a dual-energy $C T$ study. Ann Rheum Dis. 2013;72:1545-8.

3. Roddy E, Muller S, Rome K, Chandratre P, Hider SL, Richardson J, et al. Foot problems in people with gout in primary care: baseline findings from a prospective cohort study. J Foot Ankle Res. 2015;8:31.

4. Rome K, Frecklington M, McNair P, Gow P, Dalbeth N. Foot pain, impairment, and disability in patients with acute gout flares: a prospective observational study. Arthritis Care Res (Hoboken). 2012:64:384-8.

5. Rome K, Erikson K, Ng A, Gow PJ, Sahid H, Williams AE. A new podiatry service for patients with arthritis. NZ Med J. 2013;126:70-7.

6. Frecklington M, Dalbeth $N$, McNair P, Gow P, Williams A, Carroll M, et al. Footwear interventions for foot pain, function, impairment and disability for people with foot and ankle arthritis: a literature review. Semin Arthritis Rheum. 2018;47:814-24.

7. Rome K, Frecklington M, McNair P, Gow P, Dalbeth N. Footwear characteristics and factors influencing footwear choice in patients with gout. Arthritis Care Res (Hoboken). 2011;63:1599-604

8. Rome K, Stewart S, Vandal AC, Gow P, McNair P, Dalbeth N. The effects of commercially available footwear on foot pain and disability in people with gout: a pilot study. BMC Musculoskelet Disord. 2013;14:278

9. Wallace SL, Robinson H, Masi AT, Decker JL, McCarty DJ, Yü T-F. Preliminary criteria for the classification of the acute arthritis of primary gout. Arthritis Rheum. 1977:20:895-900.

10. Wolfe F, Michaud K, Pincus T. Development and validation of the health assessment questionnaire II: a revised version of the health assessment questionnaire. Arthritis Rheum. 2004:50:3296-305.

11. Singh JA, Taylor WJ, Simon LS, Khanna PP, Stamp LK, McQueen FM, et al. Patient-reported outcomes in chronic gout: a report from OMERACT 10. J Rheumatol. 2011;38:1452-7.

12. McNair PJ, Prapavessis H, Collier J, Bassett S, Bryant A, Larmer P. The lowerlimb tasks questionnaire: an assessment of validity, reliability, responsiveness, and minimal important differences. Arch Phys Med Rehabil. 2007;88:993-1001 
13. Helliwell P, Reay N, Gilworth G, Redmond A, Slade A, Tennant A, et al. Development of a foot impact scale for rheumatoid arthritis. Arthritis Rheum. 2005;53:418-22.

14. Rome K, Survepalli D, Sanders A, Lobo M, McQueen FM, McNair P, et al. Functional and biomechanical characteristics of foot disease in chronic gout: a case-control study. Clin Biomech (Bristol, Avon). 2011;26:90-4.

15. Stewart S, Dalbeth N, Otter S, Gow P, Kumar S, Rome K. Clinically-evident tophi are associated with reduced muscle force in the foot and ankle in people with gout: a cross-sectional study. J Foot Ankle Res. 2017;10:25.

16. Aati O, Taylor WJ, Horne A, Dalbeth N. Toward development of a tophus impact questionnaire, a qualitative study exploring the experience of people with tophaceous gout. Clin Rheumatol. 2014;20:251-5.

17. Miller JE, Nigg BM, Liu W, Stefanyshyn DJ, Nurse MA. Influence of foot, leg and shoe characteristics on subjective comfort. Foot Ankle Int. 2000;21:759-67.

18. Tatlock S, Rudell K, Panter C, Arbuckle R, Harrold LR, Taylor WJ, et al. What outcomes are important for gout patients? In-depth qualitative research into the gout patient experience to determine optimal endpoints for evaluating therapeutic interventions. Patient. 2017;10:65-79.

Ready to submit your research? Choose BMC and benefit from:

- fast, convenient online submission

- thorough peer review by experienced researchers in your field

- rapid publication on acceptance

- support for research data, including large and complex data types

- gold Open Access which fosters wider collaboration and increased citations

- maximum visibility for your research: over $100 \mathrm{M}$ website views per year

At $\mathrm{BMC}$, research is always in progress.

Learn more biomedcentral.com/submissions 